\title{
Measurements of transverse momentum in semi-inclusive deep-inelastic scattering at CLAS
}

\author{
K.A. Griffioen*i \\ College of William \& Mary and Helmholtz-Institut Mainz. \\ Dept. of Physics, PO Box 8795, Williamsburg, VA, USA \\ Johannes Gutenberg-Universität, Johann-Joachim-Becher-Weg 36, D-55128 Mainz, Germany \\ E-mail: griffephysics.wm.edu
}

\begin{abstract}
With mounting experimental evidence that only a small fraction of the proton's spin comes from the spins of its quarks and gluons, the quest for orbital angular momentum has begun. The parton distributions relevant to this depend on transverse quark momenta. Recent CLAS semi-inclusive deep-inelastic scattering measurements probe these new transverse-momentum-dependent parton distributions using longitudinally polarized beams and targets and detecting $\pi^{+}, \pi^{-}$and $\pi^{0}$ in the final state.
\end{abstract}

50th International Winter Meeting on Nuclear Physics - Bormio2012,

23-27 January 2012

Bormio, Italy

*Speaker.

${ }^{\dagger}$ For the CLAS Collaboration at Jefferson Lab 
The more we learn about the proton the less we seem to understand it. Although discovery of its large anomalous magnetic moment was the initial indication that the proton was not a fundamental particle, its rms charge radius was only first measured in the $1950 \mathrm{~s}$ to be $0.8 \mathrm{fm}$ using elastic electron scattering [1]. In the half-century thereafter, such measurements have been refined to yield a value of $0.879 \pm 0.008 \mathrm{fm}$ [2]. However, recent measurements of the Lamb shift in muonic hydrogen have produced a very precise value of $0.84184 \pm 0.00067 \mathrm{fm}$ [3], which is inconsistent with the electron scattering results. This Size Crisis is currently unresolved. In 1983 the European Muon Collaboration (EMC) reported [4] that the deep-inelastic structure function $F_{2}$ for the nucleons within an atomic nucleus, when compared to $F_{2}$ for the deuteron, was depleted at intermediate values of quark momentum fraction $x$, suggesting that the nucleons inside a nucleus have modified quark distributions. The EMC effect has since been rigorously measured, but the effect remains unexplained. In 1988 the EMC again surprised the world by showing data indicating that the proton's spin does not seem to come from the quark spins [5]. This precipitated the Spin Crisis. The best evidence to date suggests that the quark and gluon spins contribute a small fraction to the total proton spin [6], leaving quark and gluon orbital angular momentum as the likely source of the proton's spin. Without an understanding of orbital angular momentum, however, the Spin Crisis remains unresolved. In this context, there is great incentive to measure the orbital angular momentum of quarks in a proton.

$$
\begin{aligned}
& \frac{d \sigma}{d x d y d \psi}=\frac{2 \alpha^{2}}{x y Q^{2}} \frac{y^{2}}{2(1-\varepsilon)}\left\{\left(F_{\mathcal{T}}\right)+\varepsilon F_{L}+S_{\|} \lambda_{e} \sqrt{1-\varepsilon^{2}} 2 x\left(g_{\left.1-\gamma^{2} g_{2}\right)}\right)\right.
\end{aligned}
$$

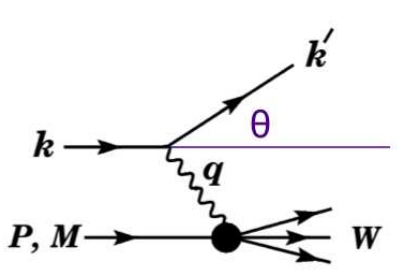

$$
\begin{aligned}
& \left.-\left|\boldsymbol{S}_{\perp}\right| \lambda_{e} \sqrt{2 \varepsilon(1-\varepsilon)} \cos \phi_{S} 2 x \gamma\left(g_{\left.1+g_{2}\right)}\right)\right\}
\end{aligned}
$$

Figure 1: Cross section and reaction diagram for inclusive deep-inelastic scattering.

Everything there is to know about a proton's partons is encoded in the Wigner functions [7],

$$
X\left(x, \xi, \mathbf{p}_{T}^{2}, \mathbf{b}_{T}^{2}, \mathbf{p}_{T} \cdot \mathbf{b}_{T} ; Q^{2}\right) .
$$

Here $x$ is the longitudinal momentum fraction of a parton, $\xi$ is half of the longitudinal momentum fraction transfered to a parton surgically removed and put back into the proton in processes such as deeply virtual Compton scattering, $\mathbf{p}_{T}$ is the transverse momentum of the parton, $\mathbf{b}_{T}$ is its transverse position, and $\mathbf{p}_{T} \cdot \mathbf{b}_{T}$ is the angular correlation between parton transverse location and momentum. The Wigner functions evolve with the 4-momentum transfer scale $Q^{2}$ as dictated by quantum chromodynamics (QCD). A Fourier transform of $\mathbf{b}_{T}$ gives the conjugate momentum $\Delta_{T}$. The Wigner functions, however, remain elusive because we are unable to measure them directly. Projections of these grand functions bring us closer to experiment. Integration over $\mathbf{p}_{T}$ gives the Generalized Parton Distributions (GPDs) $H\left(x, \xi, \Delta_{T}^{2}\right)$. Integration of $H$ over $x$ yields the proton form factors $F\left(\Delta_{T}^{2}\right)$. Instead, setting $\Delta_{T}=0$ in $H$ gives the colinear parton distribution functions 
(PDFs) $q(x)$. In addition, the Wigner functions, when evaluated at $\xi=0$ and $\Delta_{T}=0$, yield the transverse momentum distributions (TMDs) $q\left(x, \mathbf{p}_{T}^{2}\right)$, which, in turn, when integrated over $\mathbf{p}_{T}^{2}$ also give $q(x)$. Of these splendid mathematical objects only the form factors can be directly measured, and were this easy, perhaps we would have already resolved the proton size crisis. In inclusive deep-inelastic scattering (DIS), we are sensitive to the colinear PDFs $q(x)$, but thanks to QCD evolution we need to measure over a large range of $Q^{2}$ in order to deconvolve $q(x)$ from the gluon distribution.

$$
\begin{aligned}
& \frac{d \sigma}{d x d y d \psi d z d \phi_{h} d P_{h \perp}^{2}}= \\
& \frac{\alpha^{2}}{x y Q^{2}} \frac{y^{2}}{2(1-\varepsilon)}\left(1+\frac{\gamma^{2}}{2 x}\right)\left\{F_{U U, T}+\varepsilon F_{U U, L}+\sqrt{2 \varepsilon(1+\varepsilon)} \cos \phi_{h} F_{U U}^{\cos \phi_{h}}\right. \\
& +\varepsilon \cos \left(2 \phi_{h}\right) F_{U U}^{\cos } 2 \phi_{h}+\lambda_{e} \sqrt{2 \varepsilon(1-\varepsilon)} \sin \phi_{h} F_{L U}^{\sin \phi_{h}} \\
& +S_{\|}\left[\sqrt{2 \varepsilon(1+\varepsilon)} \sin \phi_{h} F_{U L}^{\sin \phi_{h}}+\varepsilon \sin \left(2 \phi_{h}\right) F_{U L}^{\sin 2 \phi_{h}}\right] \\
& +S_{\|} \lambda_{e}\left[\sqrt{1-\varepsilon^{2}} F_{L L}+\sqrt{2 \varepsilon(1-\varepsilon)} \cos \phi_{h} F_{L L}^{\cos \phi_{h}}\right] \\
& +\left|S_{\perp}\right|\left[\sin \left(\phi_{h}-\phi_{S}\right)\left(F_{U T, T}^{\sin \left(\phi_{h}-\phi_{S}\right)}+\varepsilon F_{U T, L}^{\sin \left(\phi_{h}-\phi_{S}\right)}\right)\right. \\
& +\varepsilon \sin \left(\phi_{h}+\phi_{S}\right) F_{U T}^{\sin \left(\phi_{h}+\phi_{S}\right)}+\varepsilon \sin \left(3 \phi_{h}-\phi_{S}\right) F_{U T}^{\sin \left(3 \phi_{h}-\phi_{S}\right)} \\
& \left.+\sqrt{2 \varepsilon(1+\varepsilon)} \sin \phi_{S} F_{U T}^{\sin \phi_{S}}+\sqrt{2 \varepsilon(1+\varepsilon)} \sin \left(2 \phi_{h}-\phi_{S}\right) F_{U T}^{\sin \left(2 \phi_{h}-\phi_{S}\right)}\right]
\end{aligned}
$$

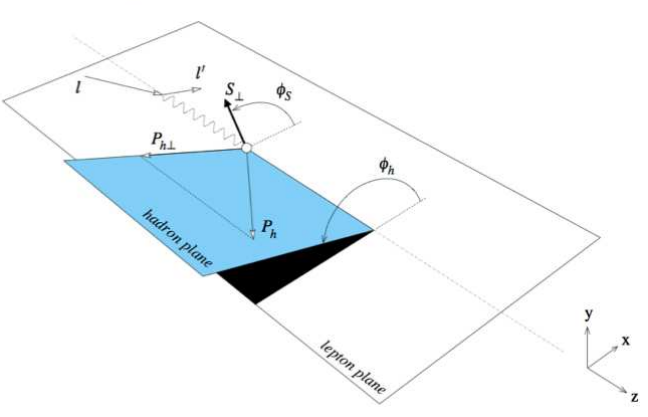

Figure 2: Cross section and reaction diagram for semi-inclusive deep-inelastic scattering.

If we are to understand orbital angular momentum, we need to press further up the chain of Wigner projections into the realms of GPDs and TMDs. At CLAS in Hall B at Jefferson Lab, we are interested in both TMDs and GPDs. The few pages that follow describe the ongoing work at CLAS to understand TMDs using longitudinally polarized beams and targets and semi-inclusive deep-inelastic scattering (SIDIS) with fragmentation producing a $\pi^{+}, \pi^{-}$or $\pi^{0}$. Let us first begin with inclusive DIS as shown in Fig. 1. An electron with 4-momentum $k$ scatters at angle $\theta$ from a target of 4-momentum $P$ and mass $M$ at rest in the lab. The scattered electron's 4-momentum is $k^{\prime}$ and the 4-momentum of the exchanged virtual photon is $q=k-k^{\prime}$. The cross section can be written in terms of $Q^{2}=-q \cdot q, v=(P \cdot q) / M, W^{2}=(P+q)^{2}, s=(k+P)^{2}, x=Q^{2} /(2 P \cdot q)$, $\varepsilon=\left[1+2\left(1+\frac{1}{\gamma^{2}}\right) \tan ^{2} \frac{\theta}{2}\right]^{-1}$, and the azimuthal angle of the lepton plane $\psi$. For a polarized beam, the helicity is given as $\lambda_{e}$. For a polarized target, the spin along the virtual photon direction is $S_{\|}$ and the component of the spin vector perpendicular to the virtual photon direction is $\mathbf{S}_{\perp}$, with $\phi_{S}$ being the angle that $\mathbf{S}_{\perp}$ makes with the lepton plane (see Fig. 2). The DIS and the SIDIS cross sections in Figs. 1 and 2 have their principal $z$ axis along the virtual photon direction. 
In the parton model

$$
\begin{aligned}
F_{T} & =2 x F_{1}=\sum_{i} e_{i}^{2} x\left[q_{i}^{\uparrow}(x)+q_{i}^{\downarrow}(x)\right], \\
F_{L} & =\left(1+\gamma^{2}\right) F_{2}-2 x F_{1}=\gamma^{2} F_{T} \rightarrow 0, \\
g_{1} & =\frac{1}{2} \sum_{i} e_{i}^{2}\left[q_{i}^{\uparrow}(x)-q_{i}^{\downarrow}(x)\right], \\
g_{2} & =-g_{1}(x)+\int_{x}^{1} \frac{d x^{\prime}}{x^{\prime}} g_{1}\left(x^{\prime}\right), \quad \text { and } \\
\gamma^{2} & =\frac{Q^{2}}{v^{2}}=\frac{4 M^{2} x^{2}}{Q^{2}},
\end{aligned}
$$

in which $q_{i}(x)$ are the quark distribution functions with spins aligned ( $\left.\uparrow\right)$ and anti-aligned $(\downarrow)$ with the proton's spin, $i$ runs over the quarks $u, \bar{u}, d, \bar{d}, s$, and $\bar{s}, e_{i}$ is the quark charge in units of the proton's charge, and $F_{1}, F_{2}$ are the more familiar unpolarized structure functions. Of course, Eqs. 2 are simplistic, since each PDF evolves with $Q^{2}$ in QCD. As experimenters, we can measure the objects $F_{L}, F_{T}, g_{1}$, and $g_{2}$ as a function of $x$ and $Q^{2}$. These are robust experimental quantities. However, their interpretation in terms of quark distribution probabilities requires a sophisticated extraction using next-to-leading order QCD fits. Despite this complication, the measured structure functions from multiple experiments give a consistent mapping of these PDFs [6].

SIDIS is naturally more complicated, and the prospects for measuring such a multi-fold differential cross section are daunting and expensive. The cross section [8] in Fig. 2 now depends on $x, Q^{2}$, and $\psi$, as before, plus the independent kinematic variables describing an observed hadron. These are $z=E_{h} / v$, the hadron energy $E_{h}$ as a fraction of the virtual photon energy $v ; \phi_{h}$, the angle between the lepton plane and the virtual photon plus hadron plane; and $P_{h \perp}$, the hadron momentum perpendicular to the virtual photon momentum. The differential $d y$ can be transformed into $d Q^{2}$, and $d \psi$ is an arbitrary rotation that integrates away, but we are left unavoidably with 5 independent variables. Each of the structure functions $\mathrm{F}$ is labeled with two subscripts, the first indicating the photon polarization state ( $\mathrm{U}$ for unpolarized and $\mathrm{L}$ for helicity $\lambda_{e}$ ), and the second indicating the target polarization ( $\mathrm{U}$ for unpolarized, $\mathrm{L}$ for polarization along the photon direction, and $\mathrm{T}$ for polarization transverse to the photon direction). This is the theoretically preferred frame. For experiments, these polarizations are measured with respect to the beam direction, which requires a frame rotation and a set of depolarization factors to transform from one to the other. The cross section in Fig. 2 was derived to sub-leading twist. This means that the structure functions $F$ in pink are the only ones that would survive at infinite $Q^{2}$ and those in blue should fade away with an additional factor of $1 / Q$. The green structure functions are the longitudinal equivalents of $F_{L}$ listed in the DIS case above and are zero in the simple parton model. To see past the overwhelming complexity of the SIDIS cross section, one has only to realize that the structure functions here too should be experimentally robust objects, independent of their interpretation, and that they can be extracted easily from measurements by a Fourier analysis in $\phi_{h}$. Because of limited statistics, the first generation of experiments at HERMES, COMPASS and JLab have typically measured various asymmetries of these structure functions, for example $A_{L U}=F_{L U} / F_{U U}$, as a function of $\phi_{h}$ and one other variable, $x, z$ or $P_{h \perp}$, summing over the rest, and extracted the appropriate sinusoidal components in $\phi_{h}$ from the angular distributions. 
In the present CLAS experiment, called EG1-DVCS, we have a longitudinally polarized $\mathrm{NH}_{3}$ target and a polarized electron beam. Therefore, we were able to explore the structure functions marked with arrows in Fig. 2.
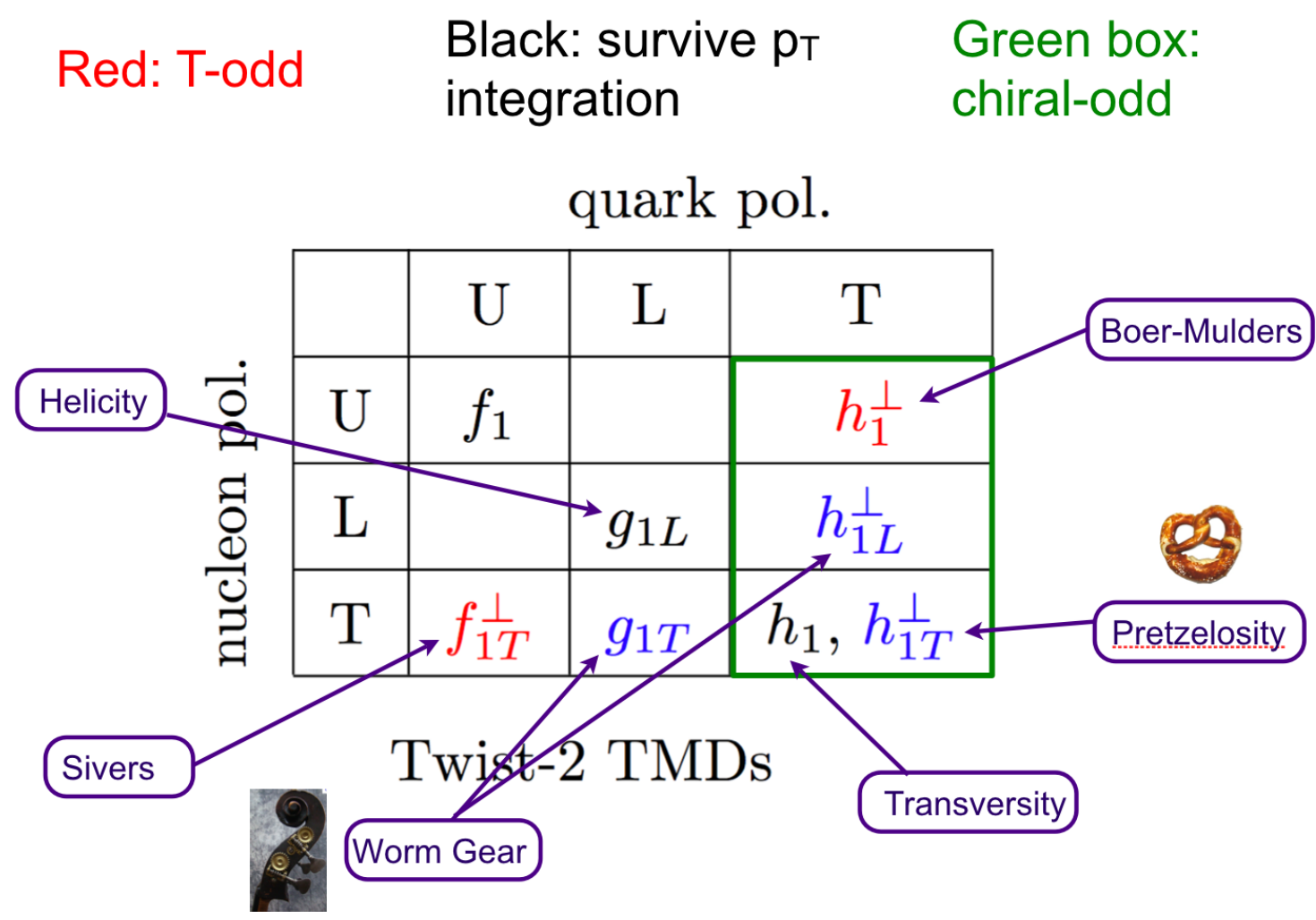

Figure 3: The periodic table of transverse momentum distributions.

In inclusive scattering, one obtains a one-dimensional description of the proton along the axis of the absorbed photon. However, a proton is a 3-dimensional object, and the quarks contained within a $0.8 \mathrm{fm}$ radius must have a component of momentum $p_{T}$ transverse to the axis of the photon. Therefore, the quark distribution functions must depend on both $x$ and $p_{T}$. Fig. 3 shows a new periodic table of these transverse momentum-dependent parton distributions. The Boer-Mulders function, $h_{1}^{\perp}\left(x, p_{T}\right)$, for example, gives the probability of finding a transversely polarized quark with momentum fraction $x$ and transverse momentum $p_{T}$ in an unpolarized proton. These TMDs have acquired interesting names such as the worm-gear functions, which contain information on quark spins that are perpendicular to the proton spin, just as a rotating worm-gear has angular momentum along two perpendicular axes. The black functions along the diagonal survive an integration over $p_{T}$. The $h$ functions are chiral-odd, which means they contain a helicity-flip that must be compensated by an analogous helicity flip in the associated fragmentation function. The red functions are naively time-reversal odd. As a group, these distributions hold information on quark spin-orbit correlations, which in one way or another may help us get to the bottom of the question of quark angular momentum in the proton. Do these objects exist? We don't yet know, but like the PDFs before them, we can measure a set of structure functions and see if they can be effectively described in terms of these TMDs. 


$$
\begin{aligned}
& \mathcal{C}[w f D]=x \sum_{a} e_{a}^{2} \int d^{2} \boldsymbol{p}_{T} d^{2} \boldsymbol{k}_{T} \delta^{(2)}\left(\boldsymbol{p}_{T}-\boldsymbol{k}_{T}-\boldsymbol{P}_{h \perp} / z\right) w\left(\boldsymbol{p}_{T}, \boldsymbol{k}_{T}\right) f^{a}\left(x, p_{T}^{2}\right) D^{a}\left(z, k_{T}^{2}\right) \\
& F_{U U, T}=\mathcal{C}\left[f_{1} D_{1}\right]=\mathcal{C}\left[g_{1 L} D_{1}-\begin{array}{l}
\text { Unpolarized } \\
\text { fragmentation function; } \\
\text { integrates to } \mathrm{D}_{1}\left(\mathrm{z}, \mathrm{Q}^{2}\right) \\
\begin{array}{l}
\text { Unpolarized structure } \\
\text { function; integrates to } \\
\mathrm{F}_{1}\left(\mathrm{x}, \mathrm{Q}^{2}\right)
\end{array}
\end{array}\right.
\end{aligned}
$$$$
\begin{gathered}
F_{U L}^{\sin 2 \phi_{h}}=\mathcal{C}\left[-\frac{2\left(\hat{\boldsymbol{h}} \cdot \boldsymbol{k}_{T}\right)\left(\hat{\boldsymbol{h}} \cdot \boldsymbol{p}_{T}\right)-\boldsymbol{k}_{T} \cdot \boldsymbol{p}_{T}}{M M_{h}} h_{1 L}^{\perp} H_{1}^{\perp}\right] \\
F_{U U}^{\cos 2 \phi_{h}}=\mathcal{C}\left[-\frac{2\left(\hat{\boldsymbol{h}} \cdot \boldsymbol{k}_{T}\right)\left(\hat{\boldsymbol{h}} \cdot \boldsymbol{p}_{T}\right)-\boldsymbol{k}_{T} \cdot \boldsymbol{p}_{T}}{M M_{h}} h_{1}^{\perp} H_{1}^{\perp}\right] \begin{array}{c}
\text { The Collins } \\
\text { function; integrates to } \\
\mathrm{g}_{1}\left(\mathrm{x}, \mathrm{Q}^{2}\right)
\end{array} \\
\begin{array}{c}
\text { fragmentation function } \\
\text { The Boer-Mulders }
\end{array} \\
\text { function }
\end{gathered}
$$

Figure 4: Leading order structure functions.

Fig. 4 shows how this works with the leading-order structure functions in the parton model (i.e. where $Q^{2}$ evolution is ignored). Here one sees explicitly the TMD $f^{a}\left(x, p_{T}^{2}\right)$ and the fragmentation function $D^{a}\left(z, k_{T}^{2}\right)$, in which $k_{T}$ is the transverse momentum arising from fragmentation. One measures a convolution $C$ of these amplitudes with a kinematic weighting factor $w$, in which $\hat{h}$ is the direction of the hadron's momentum. The delta function ensures that the final hadron's transverse momentum $P_{h \perp}$ comes from the intrinsic transverse momenta $p_{T}$ and $k_{T}$. The familiar DIS fragmentation functions now acquire a $k_{T}$-dependence, and a new chiral-odd fragmentation function, $H_{1}^{\perp}$, called the Collins function, needs to be paired with the $h$ TMDs in the right-hand column of Fig. 3. The expectation is that these fragmentation functions are universal, showing up in various structure functions.

Fig. 5 shows the full catalog of the structure functions we are able to measure at CLAS, including the leading (pink) and sub-leading-twist functions (blue). In this case, the relative simplicity of the leading-twist structure functions, with a single TMD appearing, is lost at sub-leading twist, with 4 different TMDs appearing. For each of the sub-leading-twist structure functions, one sees the explicit $1 / Q$ factor. The TMDs and fragmentation functions themselves are indicated as leading (pink) or sub-leading (blue) twist. Clearly, all the combinations of sub-leading TMDs with sub-leading fragmentation functions are ignored in this formalism. Several things could happen with these sub-leading-twist functions: 1) experiments could find them to be zero, 2) experiments might eventually have a large enough lever arm to see the $1 / Q$ dependence, although this will not be possible anytime soon, or 3 ) one of the 4 terms might dominate. The first term certainly receives the largest weight, since $M_{h}<<M$, at least for detected pions, but if either the TMD or the frag- 


$$
\begin{gathered}
F_{U U, T}=\mathcal{C}\left[f_{1} D_{1}\right] \quad F_{U U, L}=0 \quad F_{U U}^{\cos 2 \phi_{h}}=\mathcal{C}\left[-\frac{2\left(\hat{\boldsymbol{h}} \cdot \boldsymbol{k}_{T}\right)\left(\hat{\boldsymbol{h}} \cdot \boldsymbol{p}_{T}\right)-\boldsymbol{k}_{T} \cdot \boldsymbol{p}_{T}}{M M_{h}} h_{1}^{\perp} H_{1}^{\perp}\right] \\
F_{U U}^{\cos \phi_{h}}=\frac{2 M}{\pi^{2}} \mathcal{C}\left[-\frac{\hat{\boldsymbol{h}} \cdot \boldsymbol{k}_{T}}{M_{h}}\left(x h H_{1}^{\perp}+\frac{M_{h}}{M} f_{1} \frac{\tilde{D}^{\perp}}{z}\right)-\frac{\hat{\boldsymbol{h}} \cdot \boldsymbol{p}_{T}}{M}\left(x f^{\perp} D_{1}+\frac{M_{h}}{M} h_{1}^{\perp} \frac{\tilde{H}}{z}\right)\right] \\
F_{L U}^{\sin \phi_{h}}=\frac{2 M}{\pi^{Q}} \mathcal{C}\left[-\frac{\hat{\boldsymbol{h}} \cdot \boldsymbol{k}_{T}}{M_{h}}\left(x e H_{1}^{\perp}+\frac{M_{h}}{M} f_{1} \frac{\tilde{G}^{\perp}}{z}\right)+\frac{\hat{\boldsymbol{h}} \cdot \boldsymbol{p}_{T}}{M}\left(x g^{\perp} D_{1}+\frac{M_{h}}{M} h_{1}^{\perp} \frac{\tilde{E}}{z}\right)\right] \\
F_{U L}^{\sin \phi_{h}}=\frac{2 M}{\pi^{Q}} \mathcal{C}\left[-\frac{\hat{\boldsymbol{h}} \cdot \boldsymbol{k}_{T}}{M_{h}}\left(x h_{L} H_{1}^{\perp}+\frac{M_{h}}{M} g_{1 L} \frac{\tilde{G}^{\perp}}{z}\right)+\frac{\hat{\boldsymbol{h}} \cdot \boldsymbol{p}_{T}}{M}\left(x f_{L}^{\perp} D_{1}-\frac{M_{h}}{M} h_{1 L}^{\perp} \frac{\tilde{H}}{z}\right)\right] \\
F_{L L}^{\sin 2 \phi_{h}}=\mathcal{C}\left[-\frac{2\left(\hat{\boldsymbol{h}} \cdot \boldsymbol{k}_{T}\right)\left(\hat{\boldsymbol{h}} \cdot \boldsymbol{p}_{T}\right)-\boldsymbol{k}_{T} \cdot \boldsymbol{p}_{T}}{M M_{h}} h_{1 L}^{\perp} H_{1}^{\perp}\right] \\
\pi^{\cos \phi_{h}}\left[\frac{2 M}{\left.F_{1 L} D_{1}\right]}\left[\frac{\hat{\boldsymbol{h}} \cdot \boldsymbol{k}_{T}}{M_{h}}\left(x e_{L} H_{1}^{\perp}-\frac{M_{h}}{M} g_{1 L} \frac{\tilde{D}^{\perp}}{z}\right)-\frac{\hat{\boldsymbol{h}} \cdot \boldsymbol{p}_{T}}{M}\left(x g_{L}^{\perp} D_{1}+\frac{M_{h}}{M} h_{1 L}^{\perp} \frac{\tilde{E}}{z}\right)\right]\right.
\end{gathered}
$$

Figure 5: Leading and sub-leading order structure functions.

mentation function is small, other terms may be more important. By measuring the left-hand side of these equations, we will eventually learn whether the right-hand side makes sense.

Fig. 6 shows a schematic diagram of the Jefferson Lab superconducting accelerator facility on the left. Polarized electrons from the injector are accelerated in the North and South Linacs, recirculated through $180^{\circ}$ arcs, and directed, in our case, to Hall B. CLAS [9] (Fig. 6, right) has a large acceptance and can detect multiple particles produced in electron scattering. In yellow one sees the cryostats for the superconducting magnets which produce a toroidal field. The detector contains three regions of drift chambers (blue), a time-of-flight plastic scintillator wall (red), forward-angle Cherenkov counters (magenta) and electromagnetic calorimeters (green). In addition, we used an inner calorimeter to enhance $\pi^{0}$ reconstruction from two photons at small angles.

This experiment ran for 128 days in three parts from February to September 2009 using a 6 $\mathrm{GeV}$ electron beam with polarization of $85 \%$ and a frozen ammonia target with average polarization around $75 \%$. The dilution factor, due to unpolarized scattering from target nitrogen and helium, as well as the entrance and exit windows, ranged from 0.08 to 0.16 depending on the SIDIS kinematics. A total of $2.9 \times 10^{10}$ triggers were recorded for an integrated beam current of $36.4 \mathrm{mC}$. About $10 \%$ of the time we ran with carbon and empty targets for calibrations and dilution corrections. The integrated luminosity in the experiment was close to $10 \mathrm{fb}^{-1}$ of which about $2 \mathrm{fb}^{-1}$ were taken with an $\mathrm{ND}_{3}$ target.

Fig. 7 shows the experimental acceptance for SIDIS events with a detected pion. We cover the range $0.1<x<0.5$ and $1<Q^{2}<4 \mathrm{GeV}^{2}$, however, there is a kinematic correlation between $x$ and $Q^{2}$, despite the large acceptance of CLAS, because electrons are obstructed at small angles 

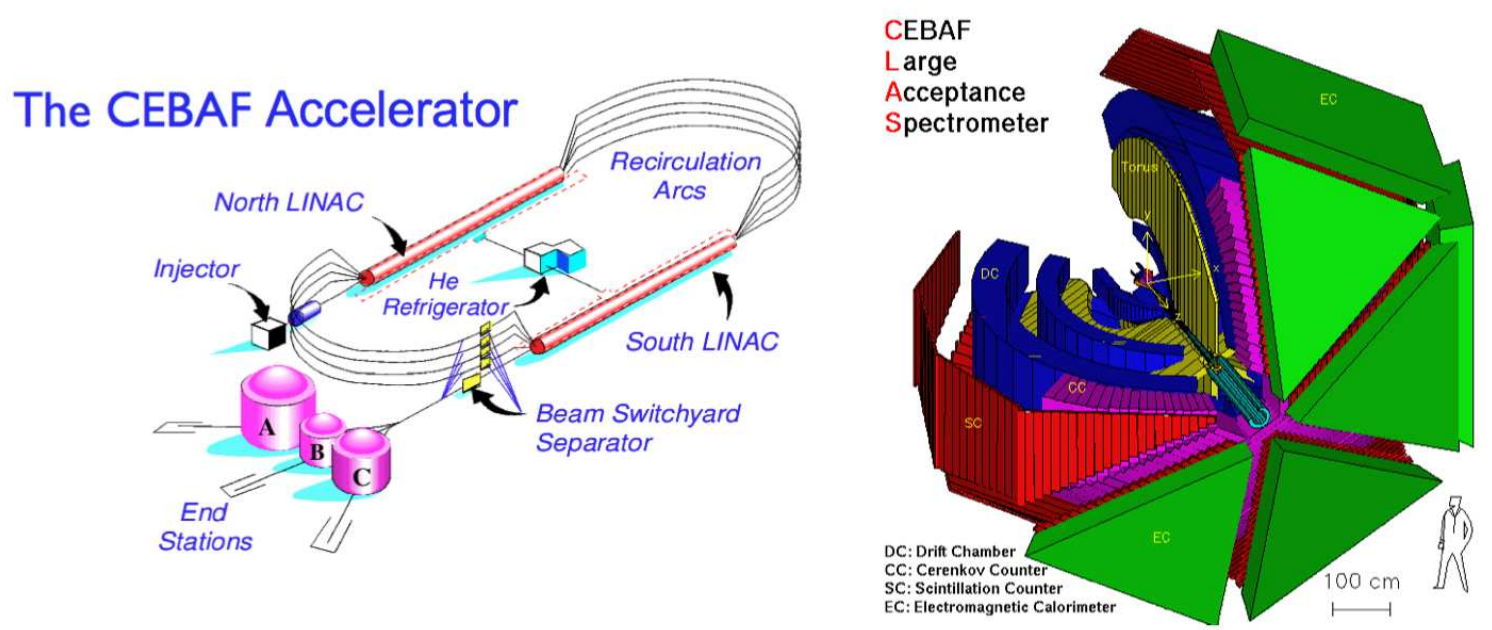

Figure 6: Schematic diagrams of the Jefferson Lab accelerator and CLAS
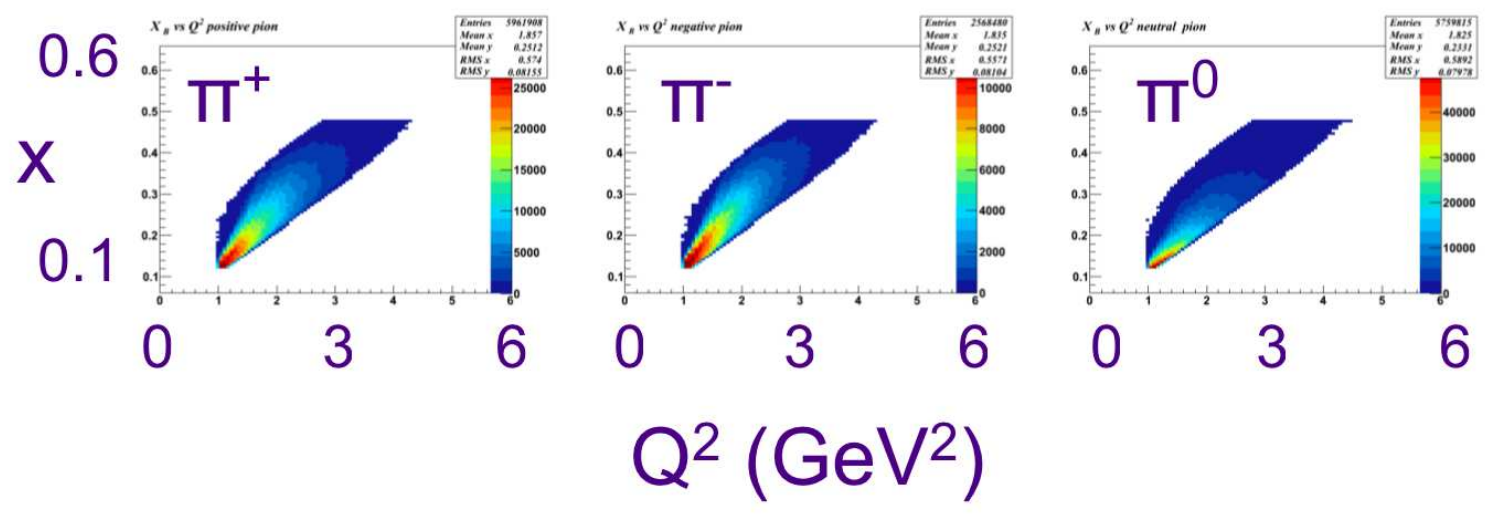

6

Figure 7: Experimental acceptance in $x$ and $Q^{2}$ for pions.

$\left(<20^{\circ}\right)$ by the inner calorimeter and at large angles $\left(>40^{\circ}\right)$ by the polarized target apparatus. Nevertheless, we have collected sufficient data to extract structure functions as a function of $x$, $P_{h \perp}$, and $\phi_{h}$. Compared to all previous measurements of this sort, we are able to produce moments of structure functions versus two variables rather than a single one. The new data increase the integrated luminosity by an order of magnitude compared to measurements taken at CLAS a decade ago [10].

Fig. 8 shows a sample of preliminary data that we have obtained. We measure asymmetries, namely the ratios of the polarized to unpolarized cross sections $A_{U L}=F_{U L} / F_{U U}, A_{L U}=F_{L U} / F_{U U}$ and $A_{L L}=F_{L L} / F_{U U}$. In this case the first subscript indicates the beam polarization and the second the target polarization along the beam direction. Data are selected with $0.4<z<0.7$ so as to avoid diffractive processes at large $z$ and target fragmentation at low $z$. All $Q^{2} \mathrm{~s}$ are summed. The result is a full $\phi_{h}$ spectrum at any of 6 values of $x$ and 9 values of $P_{h \perp}$ for $\pi^{+}, \pi^{-}$and $\pi^{0}$ SIDIS events. The two-dimensional array of plots in Fig. 8 shows the specific example of $A_{L U}$ for $\pi^{+}$in red. Nine such grids exist for the three spin configurations UL, LU and LL, and the three pion types. The 


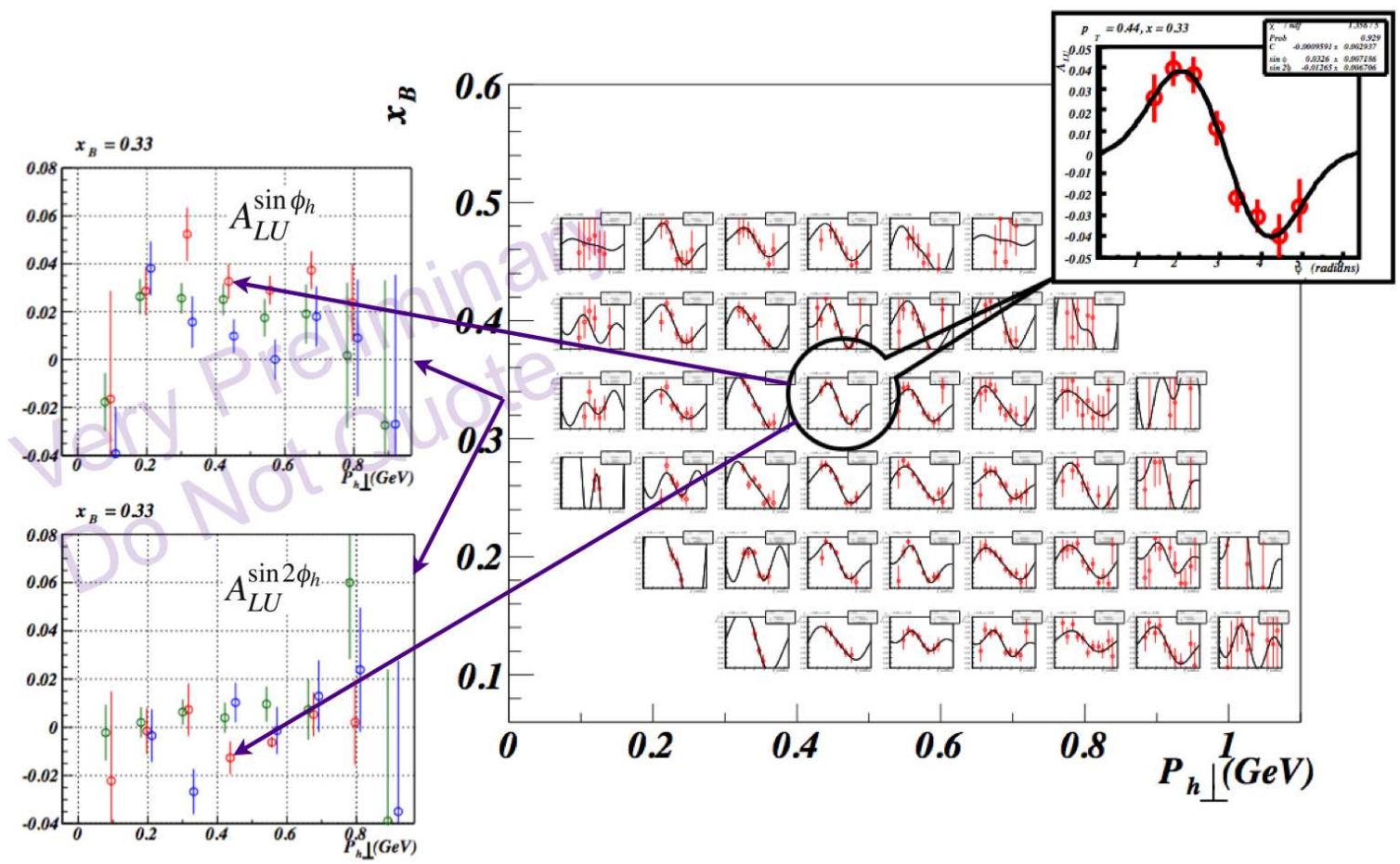

Figure 8: Asymmetries $A_{L U}$ as a function of azimuthal pion angle $\phi_{h}$ for 44 bins in $x$ and $P_{h \perp}$. Fits to these spectra of the form $A_{L U}=\sin \phi_{h} A_{L U}^{\sin \phi_{h}}+\sin 2 \phi_{h} A_{L U}^{\sin 2 \phi_{h}}$ yield one point in each of the graphs to the left. Red/green/blue corresponds to $\pi^{+} / \pi^{0} / \pi^{-}$.

$\phi_{h}$ distributions in all cases are consistent with the sinusoidal variations expected in Fig. 2. In this particular example, the $\phi_{h}$ distributions are fit to $A_{L U}=\sin \phi_{h} A_{L U}^{\sin \phi_{h}}+\sin 2 \phi_{h} A_{L U}^{\sin 2 \phi_{h}}$ with the $\sin \phi_{h}$ and $\sin 2 \phi_{h}$ moments used as fit parameters. The plots to the left in Fig. 8 summarize the results for the row indicated by $x=0.33$, and the arrows point directly to the red points that come from the circled $\phi_{h}$ plot. The upper-left graph shows $A_{L U}^{\sin \phi_{h}}$ and the lower-left graph shows $A_{L U}^{\sin 2 \phi_{h}}$. Also included in these plots are the equivalent results for $\pi^{-}$(blue) and $\pi^{0}$ (green). With a precision of about 0.01 , we can clearly see deviations from zero in these asymmetries. This is quite interesting because $A_{L U}^{\sin \phi_{h}}$ is sub-leading twist, which is presumably sensitive to the $e\left(x, p_{T}\right)$ TMD (see Fig. 5). However, $H_{1}^{\perp}$ is expected to be small for the $\pi^{0}$, and non-zero values in this case might indicate a contribution from $g^{\perp}$. No structure function $F_{L U}^{\sin 2 \phi_{h}}$ appears at leading or sub-leading twist in Fig. 2. Thus, $A_{L U}^{\sin 2 \phi_{h}}$ is expected to be zero. The data seem to be consistent with this assumption within statistics.

The analysis of these data, along with measured LL and UL asymmetries, is ongoing. Clearly the general framework for TMDs seems to work. With an order of magnitude more data than ever before, we are now able to produce multi-dimensional spectra that can be used to refine models of TMDs. As this field continues to develop it will rely on complementary experiments from $e^{+} e^{-}$ colliders, which can help define the fragmentation functions, as well as Drell-Yan experiments which are sensitive only to convolutions of two TMDs [11]. Hence, a combination of these three techniques will provide interesting input into the proton's spin structure for many years to come. 


\section{References}

[1] E.E. Chambers and R. Hofstadter, Phys. Rev. 103, 1454 (1956).

[2] J. C. Bernauer et al. [A1 Collaboration], Phys. Rev. Lett. 105, 242001 (2010) [arXiv:1007.5076 [nucl-ex]].

[3] R. Pohl et al., Nature 466, 213 (2010).

[4] J. J. Aubert et al. [European Muon Collaboration], Phys. Lett. B 123, 275 (1983).

[5] J. Ashman et al. [European Muon Collaboration], Phys. Lett. B 206, 364 (1988).

[6] D. de Florian, R. Sassot, M. Stratmann and W. Vogelsang, Phys. Rev. D 80, 034030 (2009) [arXiv:0904.3821 [hep-ph]].

[7] C. Lorce and B. Pasquini, Phys. Rev. D 84, 014015 (2011) [arXiv:1106.0139 [hep-ph]].

[8] A. Bacchetta, M. Diehl, K. Goeke, A. Metz, P. J. Mulders and M. Schlegel, JHEP 0702, 093 (2007) [hep-ph/0611265].

[9] B. A. Mecking et al. [CLAS Collaboration], Nucl. Instrum. Meth. A 503, 513 (2003).

[10] H. Avakian et al. [The CLAS Collaboration], Phys. Rev. Lett. 105, 262002 (2010) [arXiv:1003.4549 [hep-ex]].

[11] S. Arnold, A. Metz and M. Schlegel, Phys. Rev. D 79, 034005 (2009) [arXiv:0809.2262 [hep-ph]]. 